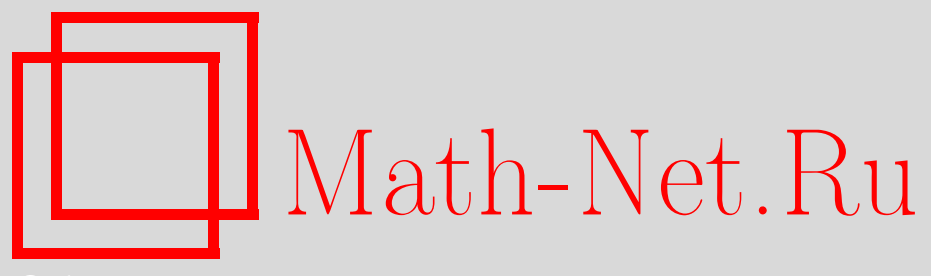

C. В. Тихонов, Полная метрика на множестве перемешивающих преобразований, УМH, 2007, том 62, выпуск 1, 209-210

DOI: https://doi.org/10.4213/rm5581

Использование Общероссийского математического портала Math-Net.Ru подразумевает, что вы прочитали и согласны с пользовательским соглашением http://www . mathnet.ru/rus/agreement

Параметры загрузки:

IP : 54.174 .149 .18

26 апреля 2023 г., 18:18:18

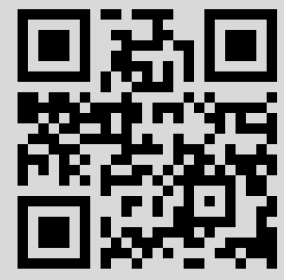




\section{Полная метрика на множестве перемешивающих преобразований}

\section{С. В. Тихонов}

В этой заметке вводится метрика на множестве перемешивающих преобразований (перемешиваний), делающая его полным сепарабельным метрическим пространством. Это открывает возможности для изучения типичных перемешиваний.

Пусть $T$ и $S$ - два обратимых сохраняющих меру преобразования пространства Лебега $(M, \Sigma, \mu), \mu(M)=1$, и $\left\{A_{i}\right\}_{i \in \mathbb{N}}-$ счетный набор множеств, порождающий $\sigma$-алгебру $\Sigma$. Будем отождествлять $T$ и $S$, если они совпадают с точностью до множества меры нуль. Преобразование $T$ называется перемешиванием, если для любых множеств $A, B \in \Sigma$ имеем $\mu\left(T^{k} A \cap B\right) \rightarrow \mu(A) \mu(B)$ при $k \rightarrow \infty$.

На множестве $\mathscr{A}$ обратимых сохраняющих меру преобразований зададим слабую топологию, предбазу окрестностей в которой образуют множества вида

$$
U\left(T, A_{i}, \varepsilon\right)=\left\{S \mid \mu\left(T A_{i} \triangle S A_{i}\right)<\varepsilon\right\} .
$$

Эта топология порождается любой из метрик

$$
d(T, S)=\sum_{i} \frac{1}{2^{i}}\left(\mu\left(T A_{i} \triangle S A_{i}\right)+\mu\left(T^{-1} A_{i} \triangle S^{-1} A_{i}\right)\right),
$$

или

$$
a(T, S)=\sum_{i, j} \frac{1}{2^{i+j}}\left|\mu\left(T A_{i} \cap A_{j}\right)-\mu\left(S A_{i} \cap A_{j}\right)\right| .
$$

Пространство $\mathscr{A}$ сепарабельно и полно относительно метрики $d(\cdot, \cdot)$.

Положим

$$
m(T, S)=d(T, S)+\sup _{k \in \mathbb{Z}} a\left(T^{k}, S^{k}\right) .
$$

Функция $m(\cdot, \cdot)$ является метрикой, как сумма двух метрик.

Топология, порожденная этой метрикой, сильнее слабой топологии пространства $\mathscr{A}$ (см. предложение ниже).

Рассмотрим множество $\mathscr{M}$ всех перемешивающих преобразований, снабженное метрикой $m$.

Лемма. Пространство $\mathscr{M}$ сепарабелъно.

ДоказАтельство. Достаточно показать, что при фиксированных $A, B \in \Sigma$ и $\varepsilon>0$ в $\mathscr{M}$ существует счетный набор множеств, покрывающий $\mathscr{M}$ и такой, что если два преобразования $T, S$ лежат в каком-либо одном из этих множеств, то $\sup _{k \in \mathbb{Z}}\left|\mu\left(T^{k} A \cap B\right)-\mu\left(S^{k} A \cap B\right)\right| \leqslant \varepsilon$.

Пусть $\mathscr{O}_{p}=\left\{T\left|\sup _{|k|>p}\right| \mu\left(T^{k} A \cap B\right)-\mu(A) \mu(B) \mid<\varepsilon / 2\right\}$. Пространство сохраняющих меру преобразований сепарабельно, и для фиксированного $p \in \mathbb{N}$ его можно покрыть счетной системой множеств $\left\{\mathscr{Q}_{l, p}\right\}_{l \in \mathbb{N}}$ таких, что

$$
P, Q \in \mathscr{Q}_{l, p} \Rightarrow \sup _{|k| \leqslant p}\left|\mu\left(P^{k} A \cap B\right)-\mu\left(Q^{k} A \cap B\right)\right|<\varepsilon .
$$

Набор множеств $\left\{\mathscr{Q}_{l, p} \cap \mathscr{O}_{p}\right\}_{p, l \in \mathbb{N}}$ искомый. Действительно, для $T, S \in \mathscr{Q}_{l, p} \cap \mathscr{O}_{p}$ имеем

$$
\begin{aligned}
\sup _{k \in \mathbb{Z}}\left|\mu\left(T^{k} A \cap B\right)-\mu\left(S^{k} A \cap B\right)\right| \leqslant \max \left\{\sup _{|k| \leqslant p}\left|\mu\left(T^{k} A \cap B\right)-\mu\left(S^{k} A \cap B\right)\right|,\right. \\
\left.\sup _{|k|>p}\left|\mu\left(T^{k} A \cap B\right)-\mu(A) \mu(B)\right|+\sup _{|k|>p}\left|\mu\left(S^{k} A \cap B\right)-\mu(A) \mu(B)\right|\right\} \leqslant \varepsilon .
\end{aligned}
$$

ПрЕДлОЖЕНиЕ. $\mathscr{M}$ - полное сепарабелъное метрическое пространство.

Работа выполнена в рамках Программы поддержки ведущих научных школ РФ (грант НШ-6849.2006.1). 
Доказательство полноты основано на том, что у фундаментальной последовательности есть предел в метрике $d(\cdot, \cdot)$ пространства $\mathscr{A}$. Непосредственно проверяется, что этот предел является перемешиванием и пределом фундаментальной последовательности в пространстве перемешиваний.

Напомним, что типичным называется свойство, выполненное для элементов счетного пересечения всюду плотных открытых подмножеств полного сепарабельного метрического пространства.

Для изучения типичных свойств перемешиваний желательно знать, когда сопряженные с преобразованием всюду плотны. В типичном случае проходят рассуждения, аналогичные тем, которые использованы в [1] при доказательстве слабого свойства Рохлина для действий счетных групп:

Теорема 1. Типичны перемешивания, сопряженные с которыми всюду плотны в $\mathscr{M}$.

Схема доказательства такова: показывается, что множество перемешиваний с требуемым свойством является всюду плотным $G_{\delta}$-множеством, затем строится пример такого перемешивания (если $\left\{T_{i}\right\}$ - всюду плотный набор перемешиваний, то примером может служить их декартово произведение).

Эта теорема не дает никаких критериев того, что сопряженные фиксированному перемешиванию всюду плотны, поэтому ее нельзя применить для доказательства всюду плотности отдельных преобразований и типичности тех или иных свойств. В этом направлении получен следующий результат.

Теорема 2. Сопряженные декартову произведению произвольных перемешиваний всюду плотны в $\mathscr{M}$. В частности, всюду плотны сопряженные любому бернуллиевскому сдвигу.

СледСтвиЕ. Следующие свойства типичны для перемешиваний: нулевая энтропия, простой спектр, дизгюнктность всех степеней.

Определения соответствующих свойств и используемые ниже факты см. в [2], [3]. Докажем, например, что перемешивания $T$, дизъюнктные своим обратным, типичны. Преобразования с этим свойством образуют $G_{\delta}$-множество в пространстве $\mathscr{A}$ (см. [4]). Поскольку естественное вложение $\mathscr{M}$ в $\mathscr{A}$ непрерывно, они образуют множество того же типа и в $\mathscr{M}$. Если $T$ - перемешивание класса $M S J(\infty)$, то перемешивание $T \times T^{2}$ дизъюнктно своему обратному. Осталось заметить, что сопряженные с $T \times T^{2}$ всюду плотны (как сопряженные с декартовым произведением).

Существуют ли перемешивания, сопряженные с которыми не всюду плотны, пока неизвестно. В этой связи возник следующий вопрос: можно ли указать перемешивание $T$ такое, что для некоторого $\varepsilon>0$ и любого множества $A, \mu(A)=\frac{1}{2}$, найдется число $k \neq 0$, для которого

$$
\left|\mu\left(T^{k} A \cap A\right)\right|>\varepsilon .
$$

Заметим, что сопряженные с таким перемешиванием не могут быть всюду плотны в $\mathscr{M}$. Следовательно, типичные перемешивания и любые декартовы произведения этим свойством не обладают. Как сообщил автору В. В. Рыжиков, он располагает доказательством того, что перемешивания с таким свойством не могут иметь конечный ранг.

\section{Список литературы}

[1] E. Glasner, J.-P. Thouvenot, B. Weiss, Bull. London Math. Soc., 38:6 (2006), 932-936. [2] И.П. Корнфельд, Я.Г. Синай, С.В. Фомин, Эргодическая теория, Наука, М., 1980. [3] V. V. Ryzhikov, Selected Russian Mathematics, 1:1 (1999), 13-24. [4] A. del Junco, Ergodic theory and dynamical systems, I (College Park, Md., 1979-1980), Progr. Math., 10, Birkhäuser, Boston, 1981, 81-89.

\section{C. В. Тихонов (S. V. Tikhonov)}

Российский государственный торгово-экономический университет

E-mail: tikhonovc@mail.ru
Представлено Д. В. Аносовым Принято редколлегией 16.06.2006 